\title{
Preparation and Evaluation of Novel Directly-Compressed Fast- Disintegrating Furosemide Tablets with Sucrose Stearic Acid Ester
}

\author{
Takuma Koseki, ${ }^{a}$ Hiraku Onishi, ${ }^{*}, a$ Yuri TAKahashi, ${ }^{a}$ Minoru UchidA, ${ }^{b}$ and Yoshiharu MachidA ${ }^{a}$ \\ ${ }^{a}$ Department of Drug Delivery Research, Hoshi University; 2-4-41 Ebara, Shinagawa-ku, Tokyo 142-8501, Japan: and \\ ${ }^{b}$ Market Development Department, Mitsubishi-Kagaku Foods Corp.; 2-11-1 Shibakoen, Minato-ku, Tokyo 105-0011, \\ Japan. Received December 12, 2008; accepted February 24, 2009
}

Fast-disintegrating tablets of furosemide (FS) were prepared by the novel direct compression method. FS, microcrystalline cellulose (MC), croscarmellose sodium (CC), xylitol (XL) and sucrose stearic acid esters (SSEs) with an hydrophilic-lipophilic balance (HLB) of 16, 15 and 11, named S1670, S1570 and S1170, were used. An FS/SSE/MC mixed powder was obtained by solvent evaporation of a suspension of MC in ethanol solution containing FS and SSE, and the resultant mixed powder was mixed with CC and XL, and directly compressed. The tablets with hardness of more than $40 \mathrm{~N}$ and disintegration time of less than $20 \mathrm{~s}$ were obtained at the addition of SSE at $0-0.5 \%(w / w)$. A tablet with S1670 at $0.1 \%(w / w)$, named TA2, dissolved faster than a commercial FS tablet, Lasix. TA2 tended to show higher plasma concentration than Lasix after intragastric administration to rats. It was demonstrated that the present direct compression using homogeneous FS/S1670/MC powder mixture could give an excellent fast-disintegrating tablet of FS.

Key words fast-disintegrating tablet; furosemide; sucrose stearic acid ester; tablet property; plasma concentration

In countries with an aging society, the number of elderly patients who have difficulty swallowing conventional tablets or capsules is increasing. ${ }^{1,2}$ Patients with oesophageal problems need oral dosage forms that can be swallowed easily. ${ }^{3-5)}$ Dosage forms such as powder or liquid are easier for these patients to swallow. However, the powder dosage form has the drawback of attaching to the oral cavity or throat, and liquid dosage forms, including emulsion or suspension, may lead to drug instability and are not necessarily convenient to carry or use. Recently, fast-disintegrating tablets have been developed to solve such problems. ${ }^{2,3,6-8)}$ As fast-disintegrating tablets can disintegrate in the oral cavity with a small amount of saliva, water is not necessary to swallow the drug, namely, patients can take it without a source of portable water.

Fast-disintegrating tablets are prepared by various techniques such as lyophilization, molding and compression. ${ }^{.-11)}$ Direct compression draws much attention because a special apparatus or container is not necessary to form the tablets, and no water or heating is required in the production. ${ }^{12)}$ However, formulation studies are essential to obtain the tablet showing appropriate disintegration rate and hardness. The disintegration time is dependent on the physicochemical characteristics and load of drugs and additives. ${ }^{12-15)}$ In the previous study, ${ }^{16}$ a fast-disintegating tablet of furosemide $(\mathrm{FS})^{17,18)}$ was developed using microcrystalline cellulose (MC) ${ }^{19,20)}$ croscarmellose sodium (CC) ${ }^{21,22)}$ xylitol (XL), ${ }^{23,24)}$ and sucrose stearic acid ester (SSE) ${ }^{25}$ ) as additives, in which the direct compression using the FS/SSE mixture prepared by evaporation of organic solvent (ethanol) ${ }^{16)}$ gave the disintegration time of less than $20 \mathrm{~s}$ and their hardness of more than $3 \mathrm{kgf}(29.4 \mathrm{~N})$ or $30 \mathrm{~N}$, which satisfied the criteria for fast-disintegrating tablets. ${ }^{26)}$ In that study, it was found that homogeneous dispersion of SSE on FS and other powders might be important to promote disintegration rate and to achieve high tablet strength. Therefore, in the present study, a novel direct compression was attempted, that is, FS/SSE/MC homogeneous powder was prepared first, then mixed with other powder, and directly compressed. Furthermore, effect of SSEs with different hydrophilic-lipophilic balance (HLB) on tablet properties was investigated. Finally, oral absorption studies were performed using rats.

\section{MATERIALS AND METHODS}

Materials Furosemide (FS) was purchased from SigmaAldrich (St. Louis, U.S.A.). Microcrystalline cellulose (MC: Avicel PH-302) and croscarmellose sodium (CC: Ac-Di-Sol) were obtained from Asahi Kasei Corp. (Tokyo, Japan). Xyliyol (XL) was purchased from Wako Pure Chemical Industries, Ltd. (Osaka, Japan). Sucrose stearic acid esters (SSEs) with HLB values of 16, 15 and 11, named S1670, S1570 and S1170, respectively, were supplied by MitsubishiKagaku Foods Corp. (Tokyo, Japan). A commercial FS tablet Lasix (20 mg), named Lasix, was purchased from SanofiAventis (Paris, France) and was used as a control. ${ }^{27)}$ All other chemicals were of reagent grade.

Animals Sprague-Dawley (SD) male rats (7 weeks old) weighing $c a .220 \mathrm{~g}$ were purchased from Tokyo Laboratory Animals Science Co., Ltd. (Tokyo, Japan), and bred on the breeding diet MF (Oriental Yeast, Tokyo, Japan) with water ad libitum at $23 \pm 1{ }^{\circ} \mathrm{C}$, relative humidity of $60 \pm 5 \%$ and a $12 \mathrm{~h}$ light-dark cycle. They were used for the experiments soon after purchase. The experimental protocol was approved by the Committee on Animal Research of Hoshi University, Japan. The animal experiments were performed in compliance with the Guiding Principles for the Care and Use of Laboratory Animals, Hoshi University, Japan.

Production of Tablets A simple direct compression tablet with no SSE, named S0 (Table 1) was prepared in the same manner as reported previously. ${ }^{16)}$ Briefly, FS, MC, $\mathrm{CC}$ and XL were sieved using a 60 mesh screen, mixed thoroughly, placed into a flat-bottomed cylinder (inner diameter $=10 \mathrm{~mm}$ ), and compressed using a cylindrical pestle with a flat end surface at $4 \mathrm{kN}$ ( $408 \mathrm{kgf})$ for $30 \mathrm{~s}$ using a Shimadzu SSP-10 A manual press (Shimadzu Corp., Kyoto, Japan). T0 
was the same as $\mathrm{S} 0$ in composition, but the preparative procedure was different. Namely, after MC, CC and XL were sieved using a 60 mesh screen, $\mathrm{MC}$ was suspended in the solution of FS in ethanol, the ethanol was evaporated at room temperature. The resultant FS/MC mixed powder was sieved with a 60 mesh screen, and mixed with $\mathrm{CC}$ and XL, and compressed in the same manner as above. TA, TB and TC tablets were produced by the same method as T0 except that MC was suspended in the ethanol solution containing FS/S1670, FS/S1570 and FS/S1170, respectively (Table 1).

Scanning Electron Microscopy FS, S1670, MC and the mixture of FS, S1670 and MC obtained by evaporation of the solvent (ethanol) were observed by scanning electron microscopy (SEM). Each powder was thinly coated with platinum using a JEOL JFC-1600 Auto Fine Coater (JEOL Ltd., Tokyo, Japan), and observed using a JEOL JSM-5600LV scanning electron microscope (JEOL Ltd., Tokyo, Japan), when micrographs were taken.

Measurement of Tablet Properties 1) Hardness: The side of the cylindrical tablet was sandwiched gently between the flat platens of a Kiya-type hardness meter (Fujiwara Scientific Co., Ltd., Tokyo, Japan), and pressed gradually. The strength immediately before crash was measured as the hardness of the tablet. 2) Disintergation: The disintegration test was performed according to the JP14 disintegration test of tablets, that is, using a disintegration tester, NT-60H (Toyama Sangyo Co., Ltd., Osaka, Japan) with water as a solvent at $37^{\circ} \mathrm{C}$. The time for the tablet or its particles to disappear completely from the tester net was measured as a disintegration time. 3) Dissolution: The JP14 second dissolution method (paddle method) was employed as a dissolution test, in which a dissolution tester, NRT-VS6P (Toyama Sangyo Co., Ltd., Osaka, Japan), was used using $500 \mathrm{ml}$ of JP14 second fluid, $\mathrm{pH} 6.8,(0.05 \mathrm{~m}$ phosphate buffer) as a solvent at $37^{\circ} \mathrm{C}$. This condition was used unless the experimental conditions were otherwise indicated. At appropriate time points, aliquot samples were withdrawn, and the concentration of FS in the supernatant was measured spectrophotometrically at $274 \mathrm{~nm}$. Immediately after each sampling, fresh JP14 second fluid was supplemented. Cumulative release was calculated from the FS concentration of the supernatant of the sample and FS taken out.

Animal Experiments The tablets, TA2 and Lasix, were used in animal studies. A portion of the tablet, corresponding to $2 \mathrm{mg}$ FS eq, was taken by cutting with a razor blade, and placed in $0.5 \mathrm{ml}$ of saline. The rats were anesthetized by an intraperitoneal (i.p.) injection of pentobarbital solution in saline at $50 \mathrm{mg} / \mathrm{kg}(2 \mathrm{ml} / \mathrm{kg})$ after they had been fasted for $20 \mathrm{~h}$. The resultant suspension was administered intragastrically to rats using a syringe with a plastic tube. Blood samples $(0.3 \mathrm{ml})$ were withdrawn via the jugular vein $0.25,0.5$, $1,2,4$ and $8 \mathrm{~h}$ after administration. Plasma was obtained by centrifugation of the blood at $3000 \mathrm{rpm}$ for $15 \mathrm{~min}$, and $100 \mu \mathrm{l}$ of the plasma was mixed with $500 \mu \mathrm{l}$ of $1 \mathrm{M}$ potassium dihydrogenphosphate. The mixture was added to $2 \mathrm{ml}$ of diethyl ether, and shaken vigorously. After the mixture was centrifuged at $3000 \mathrm{rpm}$ for $10 \mathrm{~min}$, the ether layer was taken. The remaining bottom layer was mixed with $2 \mathrm{ml}$ of fresh ethyl ether, and shaken vigorously, and the ether layer was taken. The obtained ether layers were combined, and dried under nitrogen gas. The resultant residue was dissolved in
$250 \mu \mathrm{l}$ of a mixture of $50 \mathrm{~mm}$ potassium dihydrogenphosphate and acetonitrile $(68: 32, \mathrm{v} / \mathrm{v})$. The solution was analyzed for the FS concentration by high performance liquid chromatography (HPLC). The HPLC system consisted of an LC-10AS pump, SPD-10A spetrophotometric detector, C-R7 chromatopac, SCL-10A system controller, SIL-10A autosampler and CTO-10A column oven (Shimadzu Corp., Japan). An Intersil octadecyl silica (ODS) $5 \mu \mathrm{m}$ column (4.6 mm inner diameter $\times 250 \mathrm{~mm}$ long, particle size $5 \mu \mathrm{m}$; GL Sciences, Inc., U.S.A.) was used as an analytical column, and the column oven was set at $30^{\circ} \mathrm{C}$. The detector was set at $330 \mathrm{~nm}$. A mixture of $50 \mathrm{~mm}$ potassium dihydrogenphosphate and acetonitrile $(68: 32, \mathrm{v} / \mathrm{v})$ was used as the mobile phase, and the flow rate was $1.0 \mathrm{ml} / \mathrm{min}$. The sample $(50 \mu \mathrm{l})$ was injected onto the column. The absolute calibration curve method was used for quantification.

Maximal plasma concentration $\left(C_{\max }\right)$, the time to reach $C_{\max }\left(T_{\max }\right)$, area under the plasma concentration-time curve $(A U C)$, mean residence time (MRT) and variance of residence time $(V R T)$ were calculated for the period of 0 to infinite by the trapezodidal rule using a program, MULTI. ${ }^{28)}$

Statistical Analysis Comparison was performed using the unpaired $t$-test, and significant difference was set as $p<$ 0.05 .

\section{RESULTS AND DISCUSSION}

Tablet Properties The tablet, S0, was prepared by the simple mixing of FS, MC, CC and XL, and subsequent direct compression. A combination of $\mathrm{MC}$ and $\mathrm{CC}$ is often used as a binder and/or disintegrant of tablets, and XL is used as a corrigent. As reported previously, ${ }^{16)}$ more XL increased the disintegration time, but the addition of XL at $20 \%(\mathrm{w} / \mathrm{w})$ or more was necessary to mask the unpleasant taste of FS. Thus, the formulation, S0, was chosen as a basic control (Table 1).

According to the previous study, ${ }^{16)}$ it was presumed that homogenous mixing of FS and SSE and their uniform dispersion in powder mixture would be important to obtain tablets displaying fast disintegration and high hardness. Therefore, in the present novel preparation, utilization of a more homogenous powder mixture was attempted. Namely, suspension of $\mathrm{MC}$ in ethanol solution containing FS and SSE and subsequent solvent evaporation were performed to obtain the homogeneous FS/SSE/MC powder. The resultant FS/SSE/MC powder mixture was mixed with $\mathrm{CC}$ and XL, sieved with a 60 mesh screen, and directly compressed to obtain the tablets (TA, TB and TC tablets). The T0 tablet was prepared in the same procedure except that no SSE was added. The powder features of FS, S1670, MC and the FS/S1670/MC mixture obtained by the solvent evaporation are shown in Fig. 1. For the FS/S1670/MC mixture, the mixture of FS and SSE was observed to cover almost the entire surface of each MC particle. This suggested that the FS, SSE and MC should be mixed very homogenously.

The disintegration test of fast-disintegrating tablets is generally performed using human volunteers or disintegration testers. Ogata et al. reported that the disintegration rate by the JP disintegration test was well-correlated with actual oral disintegration rate for various fast-disintegration tablets. ${ }^{29}$ ) Therefore, in the present study, the disintegration rate ob- 
Table 1. Formulations of Tablets Produced by Simple Direct Compression and by Novel Direct Compression Using SSE

\begin{tabular}{|c|c|c|c|c|c|c|c|c|c|c|c|c|c|}
\hline Component & S0 & T0 & TA1 & TA2 & TA3 & TA4 & TA5 & TB1 & TB2 & TB3 & TC1 & $\mathrm{TC} 2$ & TC3 \\
\hline FS (mg) & 20 & 20 & 20 & 20 & 20 & 20 & 20 & 20 & 20 & 20 & 20 & 20 & 20 \\
\hline $\mathrm{MC}(\mathrm{mg})$ & 134 & 134 & 134 & 134 & 134 & 134 & 134 & 134 & 134 & 134 & 134 & 134 & 134 \\
\hline CC (mg) & 6 & 6 & 6 & 6 & 6 & 6 & 6 & 6 & 6 & 6 & 6 & 6 & 6 \\
\hline XL (mg) & 40 & 40 & 40 & 40 & 40 & 40 & 40 & 40 & 40 & 40 & 40 & 40 & 40 \\
\hline $\mathrm{S} 1670(\mathrm{mg})$ & - & - & 0.1 & 0.2 & 1 & 2 & 4 & - & - & - & - & - & - \\
\hline $\mathrm{S} 1570(\mathrm{mg})$ & - & - & - & - & - & - & - & 0.1 & 0.2 & 1 & - & - & - \\
\hline $\mathrm{S} 1170(\mathrm{mg})$ & - & - & - & - & - & - & - & - & - & - & 0.1 & 0.2 & 1 \\
\hline
\end{tabular}

S0: Tablet produced by direct compression after mixing each powder. T0: Tablet produced by direct compression after mixing CC, XL and the FS/MC mixture obtained by evaporation of solvent (ethanol). TA: Tablet produced by direct compression after mixing CC, XL and the FS/S1670/MC mixture obtained by evaporation of solvent (ethanol). TB: Tablet produced by direct compression after mixing CC, XL and the FS/S1570/MC mixture obtained by evaporation of solvent (ethanol). TC: Tablet produced by direct compression after mixing CC, XL and the FS/S1170/MC mixture obtained by evaporation of solvent (ethanol).

A

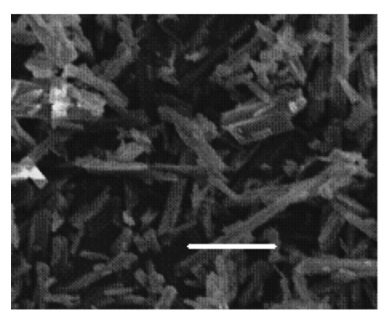

C

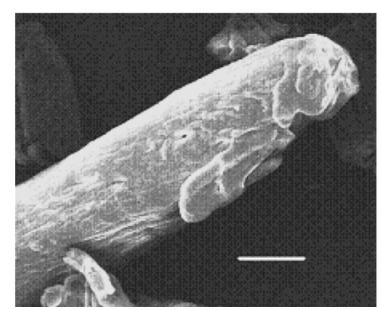

B

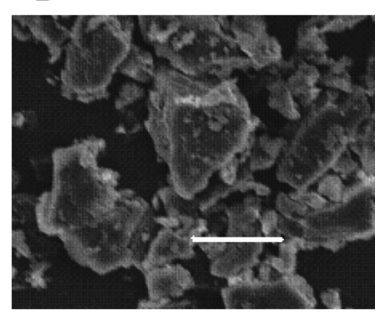

D

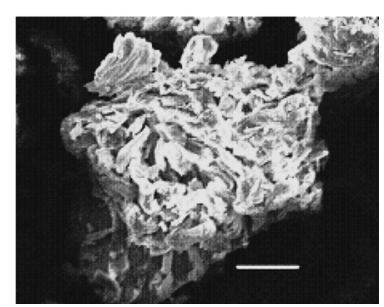

Fig. 1. SEM Pictures of FS (A), S1670 (B), MC (C) and FS/SSE/MC Mixed Powder Obtained by Solvent Evaporation (D)

The length of each white bar is $10 \mu \mathrm{m}$.

tained by the JP14 disintegration test was considered to be useful for the evaluation of the disintegration rate of each tablet. The disintegration time of commercial fast-disintegrating tablets are generally less than $20 \mathrm{~s},{ }^{26)}$ because patients feel no concern about the intraoral disintegration time of less than $20 \mathrm{~s}$. In addition, hardness is important, because the tablets have to be packed and carried without breaking. Reportedly, hardness of more than $3 \mathrm{kgf}(29.4 \mathrm{~N})$ or $30 \mathrm{~N}$ is necessary in order to ensure that they can be automatically packed or taken out of a press-through package without breaking.

The basic tablet, S0, showed slightly greater hardness than $30 \mathrm{~N}$, but the disintegration time was over $40 \mathrm{~s}$ (Fig. 2). A tablet, T0, which was prepared by the novel preparation but without using SSE, displayed high hardness of nearly $60 \mathrm{~N}$ and a good disintegration time, which was slightly less than 20 s (Fig. 2). Although S0 and T0 had the same composition, their tablet properties were quite different. Homogeneous distribution of binder was reported to be important to enhance the tablet strength, ${ }^{30}$ ) which might be true in the present study. T0, produced using the mixed powder obtained by the homogeneous treatment, exhibited high hardness and fast disintegration rate. Homogeneous distribution of FS and MC in T0 might facilitate the contact among the particles and en-
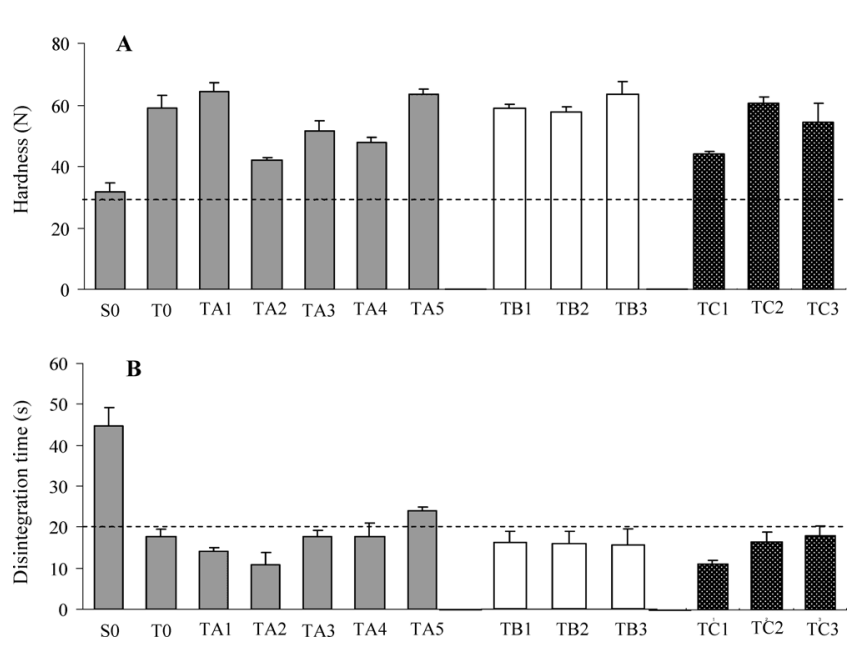

Fig. 2. Hardness (A) and Disintegration Time (B) of S0, T0, TA, TB and TC Tablets

The results are expressed as the mean \pm S.D. $(n=4$ for A; $n=6$ for B).

hance capillary absorption for solvent permeation, though the detailed mechanism remained to be solved. This consideration was suggested for TA, TB and TC tablets. The tablets, TA1-TA4, containing S1670 showed a disintegration time of less than $20 \mathrm{~s}$, and had hardness of more than $40 \mathrm{~N}$. In particular, TA2 exhibited the fastest disintegration time of approximately $10 \mathrm{~s}$, which was more acceptable for a fastdisintegrating tablet. TA2 was regarded as the best tablet, and used for the following experiments. TB and TC tablets were produced using S1570 and S1170, respectively, instead of S1670. All TB and TC tablets had hardness of more than $40 \mathrm{~N}$, and their disintegration time was less than $20 \mathrm{~s}$. TB3 and $\mathrm{TC} 1$ showed the fastest disintegration rate in TB and TC tablets, respectively, chosen as the best TB and TC tablets, respectively, and used for dissolution studies. These results demonstrated that the tablets of good quality could be obtained by the novel direct compression, and that the addition of SSEs could further improve the tablet characteristics.

In addition to hardness and the disintegration rate, dissolution is also very important for fast-disintegrating tablets, because it is directly related to efficacy. In the dissolution test, tablets of good quality, TA2, TB3 and TC1 were chosen, and T0 and Lasix were used as control tablets. Their dissolution profiles are shown in Fig. 3. The 90\% release time was approximately 4, 10 and $16 \mathrm{~min}$ for TA2, Lasix and T0, respectively. TB3 and TC1 exhibited slower dissolution rates. At 
Table 2. Pharmacokinetic Parameters after Oral Administration of Aqueous Suspension of TA2 and Lasix

\begin{tabular}{cccccc}
\hline \hline Formulation & $C_{\max }(\mu \mathrm{g} / \mathrm{ml})$ & $T_{\max }(\mathrm{h})$ & AUC $(0-\infty)(\mu \mathrm{g} \cdot \mathrm{h} / \mathrm{ml})$ & MRT $(0-\infty)(\mathrm{h})$ & $V R T(0-\infty)\left(\mathrm{h}^{2}\right)$ \\
\hline TA2 & $1.13 \pm 0.23$ & $0.33 \pm 0.08$ & $8.37 \pm 2.36$ & $10.94 \pm 4.12$ & $159.10 \pm 111.80$ \\
Lasix & $0.81 \pm 0.33$ & $0.58 \pm 0.22$ & $4.13 \pm 0.47$ & $6.54 \pm 0.57$ & $47.08 \pm 5.00$ \\
\hline
\end{tabular}

The results are expressed as the mean \pm S.E. $(n=3)$.

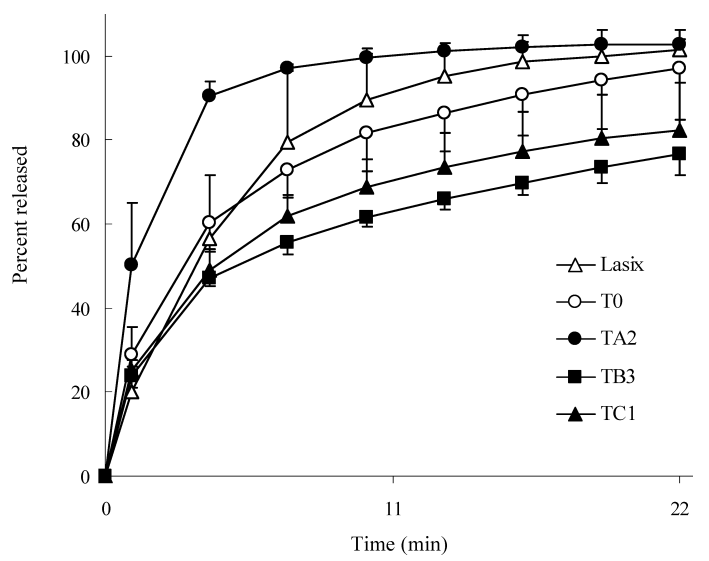

Fig. 3. Dissolution Profiles of FS for T0, Lasix, TA2, TB3 and TC1 in JP14 Second Fluid at $37^{\circ} \mathrm{C}$

The results are expressed as the mean \pm S.D. $(n=3)$.

22 min after the start of the release test, TB3 and TC1 released $77 \%$ and $82 \%$, respectively. As to the dissolution for TB2 and TC2 (data not shown), their $90 \%$ release time were 7 and $22 \mathrm{~min}$, respectively, longer than that of TA2. Thus, TA2 exhibited the fastest dissolution of FS. S1670 was considered to be most adequate in terms of the promotion of dissolution rate, probably due to its high wettability.

TA2 and Lasix were administered intragastrically at a dose of $2 \mathrm{mg}$ FS eq per rat $(9.1 \mathrm{mg} \mathrm{FS} \mathrm{eq} / \mathrm{kg})$ after their disintegration in saline. The plasma levels of FS were higher in TA2 than in Lasix throughout the observation period (Fig. 4), but the plasma levels were not significantly different between TA2 and Lasix at each point $(p>0.05)$. The pharmacokinetic parameters are described in Table 2. The $C_{\max }$ was 1.4 times larger in TA2 than in Lasix, while the $T_{\max }$ was shorter in TA2, 0.57 times that in Lasix. The $A U C$ was approximately twice larger in TA2 than in Lasix. The $M R T$ and $V R T$ were also greater in TA2 than in Lasix. However, there was no significant difference in each pharmacokinetic parameter between TA2 and Lasix $(p>0.05)$. When the dissolution study was investigated using JP14 first fluid (diluted $\mathrm{HCl}$ aqueous solution containing $0.2 \% \mathrm{NaCl}, \mathrm{pH} 1.2$ ) for $\mathrm{TA} 2$ and $\mathrm{FS}$ powder, TA2 and FS powder released $16 \%$ and $3 \%$ at $1 \mathrm{~h}$, respectively, and $30 \%$ and $24 \%$ at $24 \mathrm{~h}$, respectively (data not shown). Reportedly in the quality tests of the generic drugs, Lasix also exhibit slow release of FS in JP14 first fluid ( $c a$. $35 \%$ release at $1 \mathrm{~h}$ ). Therefore, the dissolution was considered to be caused more easily in the small intestine. Generally, fast-disintegrating tablets are expected to pass through the stomach fast. Furthermore, considering that a major absorption site of FS is the stomach and upper parts of small intestine ${ }^{31)}$ rapid dissolution is important for effective absorption from the FS fast-disintegrating tablets. As TA2 exhibited faster dissolution rate, higher plasma levels were considered to be achieved in TA2 than in Lasix. The plasma FS

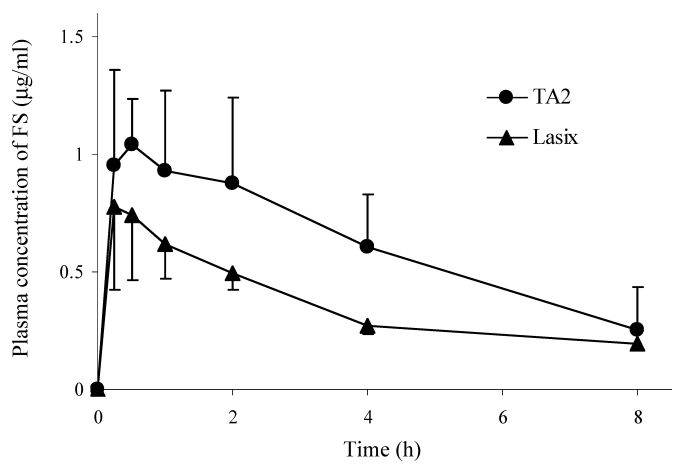

Fig. 4. Plasma Concentration-Time Profiles of FS after Intragastrical Administration of Aqueous Suspension of TA2 and Lasix at a Dose of $2 \mathrm{mg}$ FS eq per Rat

The error bar was not shown if it was smaller than a symbol size. The results are expressed as the mean \pm S.E. $(n=3)$.

level of more than $0.2 \mu \mathrm{g} / \mathrm{ml}$ is necessary to obtain sufficient diuretic effect for human. ${ }^{32,33)}$ Reportedly, such a plasma level was completed immediately after oral administration of Lasix, and lasted for several hours. ${ }^{32)}$ As Fig. 4 indicated that TA2 exhibited higher plasma concentration than Lasix, TA2 was expected to act better than Lasix.

Thus, in this study, a novel preparative method was developed and evaluated to obtain fast-disintegrating tablets. Namely, homogeneous FS/SSE/MC mixed powder was produced first, then mixed with CC and XL, and compressed directly. The obtained tablets showed good hardness of more than $4 \mathrm{~kg}$ and suitable disintegration time of less than $20 \mathrm{~s}$. The tablet prepared with the addition of S1670 at $0.1 \%$ (w/w) had excellent tablet properties for hardness and disintegration rate, exhibited faster release of FS in JP14 second fluid than Lasix, and tended to show higher plasma concentration as compared with Lasix. It is suggested that the tablet prepared by the present novel compression using SSEs, in particular, S1670, should be useful as a fast-disintegrating tablet of FS. Industrial production, actual oral disintegration rate and oral feeling might be major future subjects.

\section{REFERENCES}

1) Honda Y., Nakano M., Jpn. J. Hosp. Pharm., 24, 533-540 (1998).

2) Mizumoto T., Masuda Y., Yamamoto T., Yonemochi E., Terada K., Int. J. Pharm., 306, 83-90 (2005).

3) Watanabe A., Hanawa T., Sugihara M., Yakuzaigaku, 54, 77-87 (1994).

4) Hanawa T., Watanabe A., Tsuchiya T., Ikoma R., Hidaka M., Sugihara M., Chem. Pharm. Bull., 43, 284-288 (1995).

5) Fukami J., Yonemochi E., Yoshihashi Y., Terada K., Int. J. Pharm., 310, 101-109 (2006).

6) Baldi F., Malfertheiner P., Digestion, 67, 1-5 (2003).

7) Loder E. W., Dowson A. J., Spierings E. L., Curr. Med. Res. Opin., 21 (Suppl. 3), S8-S12 (2005).

8) Moen M. D., Keating G. M., Drugs, 66, 883-890 (2006).

9) Seager H., J. Pharm. Pharmacol., 50, 375-382 (1998). 
10) Corveleyn S., Remon J. P., Drug Dev. Ind. Pharm., 25, 1005-1013 (1999).

11) Fu Y., Yang S., Jeong S. H., Kimura S., Park K., Crit. Rev. Ther. Drug Carrier Syst., 21, 433- 476 (2004).

12) Rawas-Qalaji M. M., Simons F. E., Simons K. J., AAPS PharmSciTech., 7, E1-E7 (2006).

13) Watanabe Y., Koizumi K., Zama Y., Kiriyama M., Matsumoto Y., Matsumoto M., Biol. Pharm. Bull., 18, 1308-1310 (1995).

14) Jin Y., Ohkuma H., Wang H., Natsume H., Sugibayashi K., Morimoto Y., Yakugaku Zasshi, 122, 989-994 (2002).

15) Fukami J., Ozawa A., Yoshihashi Y., Yonemochi E., Terada K., Chem. Pharm. Bull., 53, 1536-1539 (2005).

16) Koseki T., Onishi H., Takahashi Y., Uchida M., Machida Y., Chem. Pharm. Bull., 56, 1384-1388 (2008).

17) Waller E. S., Crismon M. L., Smith R. V., Bauza M. T., Doluisio J. T., Biopharm. Drug Dispos., 9, 211-218 (1988).

18) Yagi N., Kiuchi T., Satoh H., Terashima Y., Kenmotsu H., Sekikawa H., Takada M., Biol. Pharm. Bull., 9, 616-622 (1996).

19) Tsai T., Wu J. S., Ho H. O., Sheu M. T., J. Pharm. Sci., 87, 117-122 (1998).

20) Siaan M., Pintye-Hódi K., Szabó-Révész P., Kása P. Jr., Erõs I., Drug Dev. Ind. Pharm., 26, 1013-1018 (2000).

21) Chen C. R., Cho S. L., Lin C. K., Lin Y. H., Chiang S. T., Wu H. L., Chem. Pharm. Bull., 46, 478-481 (1998).
22) Sallam E., Ibrahim H., Dahab R. A., Shubair M., Khalil E., Drug Dev. Ind. Pharm., 24, 501-507 (1998).

23) Suzuki H., Onishi H., Hisamatsu S., Masuda K., Takahashi Y., Iwata M., Machida Y., Int. J. Pharm., 278, 51—61 (2004).

24) Mäkinen K. K., Saag M., Isotupa K. P., Olak J., Nõmmela R., Söderling E., Mäkinen P. L., Caries Res., 39, 207-215 (2005).

25) Aoshima H., Miyagisnima A., Nozawa Y., Sadzuka Y., Sonobe T., Int. J. Pharm., 293, 25-34 (2005).

26) Terashi M., J. Kagoshima City Med. Assoc., 43, 20 (2004).

27) Meyer B. H., Müller F. O., Hundt H. K., Grigoleit H. G., Hajdu P., Heptner W., S. Afr. Med. J., 59, 891-893 (1981).

28) Yamaoka K., Tanigawara Y., Nakagawa T., Uno T., J. Pharmacobiodyn., 4, 879-885 (1981).

29) Ogata K., Takamura N., Kashiwagi S., Hamada R., Kodama H., Arimori K., Jpn. J. Pharm. Health Care Sci., 27, 553-558 (2001).

30) Gavrilov A. S., Gusel'nikova E. V., Ptrov A. V., Pharmaceut. Chem. J., 38, 41- 44 (2004).

31) Säkkinen M., Tuononen T., Jürjenson H., Veski P., Marvola M., Eur. J. Pharm. Sci., 19, 345-353 (2003).

32) Nihon Generic Co., Ltd., "Interview Form: Furosemide Tablets $20 \mathrm{mg}$ JG," 2007, p. 9 .

33) Kyorin Rimedio Co., Ltd., "Interview Form: Furosemide Tablets 40 mg Mita," 2008, p. 14 